Aim of the study: The spectrum of pulmonary infections in patients with lung cancer is wide, and tools for target-oriented infection control measures are necessary. In this retrospective study we report the microbiological spectrum of pneumonia (based on the results of sputum culture) in a case series of Turkish patients with lung malignancies.

Material and methods: Between 2010 and 2011 a total of 119 patients (111 males and 8 females, mean age: $59.8 \pm 9.6$ years) with lung cancer and pneumonia were identified at the Department of Medical Oncology of two Turkish Universities (Uludag University, Bursa and Cumhuriyet University, Sivas). Expectorated sputum samples were collected in sterile specimen containers and processed immediately in the hospital bacteriology laboratory. Results: Of the 119 study patients, $92(77.3 \%)$ had positive isolates from sputum cultures. The most frequent isolate from the sputum of lung cancer patients with pneumonia was Aspergillus fumigatus $(n=22)$, followed by Haemophilus influenzae $(n=13)$ and Pseudomonas aeruginosa $(n=12)$. The likelihood of having a positive Aspergillus fumigatus sputum culture was significantly and independently associated with febrile neutropaenia $(\mathrm{OR}=1.32,95 \% \mathrm{Cl}$ : $1.17-3.68, p<0.05)$ and the development of pneumonia within the first 10 days of chemotherapy initiation (OR $=1.78,95 \% \mathrm{Cl}: 1.37-4.12, p<0.01$ ). Conclusions: We conclude that Aspergillus fumigatus was the most frequent isolate, but the high diversity of pathogens clearly challenges the empirical use of antimicrobial drugs.

Key words: lung cancer, pneumonia, Aspergillus fumigatus, febrile neutropaenia.

Contemp Oncol (Pozn) 2016; 20 (1): 63-66 DOI: $10.5114 /$ wo. 2016.58502

\section{Retrospective analysis of the microbiological spectrum of pneumonia in Turkish patients with lung cancer}

\author{
Nilüfer Avcı' ${ }^{1}$ Mustafa Hartavi², Turgut Kaçan³ ${ }^{3}$, Murat Bayındır², \\ Mustafa $\mathrm{AvCl}^{2}$, Cüneyt Özakın ${ }^{4}$, Aynur Engin ${ }^{5}$, Adem Deliqönüll', \\ Turkkan Evrensel ${ }^{6}$, Halis Akalın ${ }^{5}$
}

${ }^{1}$ Department of Medical Oncology, Balıkesir State Hospital, Balıkesir, Turkey ${ }^{2}$ Department of Internal Medicine, Uludag University, Bursa, Turkey

${ }^{3}$ Department of Medical Oncology, Cumhuriyet University, Sivas, Turkey ${ }^{4}$ Department of Medical Microbiology, Uludag University, Bursa, Turkey

${ }^{5}$ Department of Infectious Diseases and Clinical Microbiology, Cumhuriyet University, Sivas, Turkey

${ }^{6}$ Department of Medical Oncology, Uludag University, Bursa, Turkey

\section{Introduction}

In Turkey, lung cancer is the most commonly diagnosed malignant disease in men and the fifth most frequently diagnosed malignancy in women [1]. Notably, lung malignancies are the leading cause of death from cancer among Turkish people aged between 45 and 70 years, as reported in Turkish Republic Ministry of Health Reports 2009 [2]. Although lung tumours exert an enormous human and economic toll, the exact causes of death for patients with lung cancer have not been completely elucidated yet. However, both tumour burden (either in the form of extensive distant metastases or primarily lung tumour burden causing respiratory failure) and infective complications (e.g. pneumonia and sepsis) are considered the leading causes of death in this patient population [3].

Patients with lung cancer are particularly prone to respiratory infections for several reasons, including immune dysregulation (both at the cellular and humoral level) caused by their cancers, use of immunosuppressive treatments (e.g. chemotherapy or corticosteroids), poor performance status or the presence of comorbidities, and changes in endogenous bacterial flora during prolonged hospitalisations [4-8].

Because the spectrum of pulmonary infections in patients with lung cancer is wide and tools for target-oriented infection control measures are necessary [9], in this retrospective study we report the microbiological spectrum of pneumonia (based on the results of sputum culture) in a case series of Turkish patients with lung malignancies. We also aimed to investigate the potential correlates of pneumonia in patients with lung tumours.

\section{Material and methods}

Study participants

Between 2010 and 2011 a total of 119 patients (111 males and 8 females, mean age: $59.8 \pm 9.6$ years) with lung cancer and pneumonia were identified at the Department of Medical Oncology of two Turkish Universities (Uludag University, Bursa and Cumhuriyet University, Sivas). The diagnosis of pneumonia was based on clinical signs and symptoms of lower respiratory tract infection and the presence of a new infiltrate on chest radiographs, which was at least segmental and was not due to any pre-existing or other known cause [10]. Community-acquired pneumonia (CAP) was defined as an 
acute infection of the pulmonary parenchyma in a patient who acquired the infection in the community, as distinguished from hospital-acquired pneumonia. The general demographic and clinical characteristics of the study participants were collected from clinical charts. The protocol followed the tenets of the Helsinki Declaration and was approved by the Institutional Review Board. Individual patient consent was obtained for entry into the database. However, our Institutional Review Board waived the need for individual patient consent for this study.

\section{Treatment of lung cancer}

Twenty-six patients (21.8\%) underwent surgical treatment (12 for palliation and 14 with curative intent). A total of 33 study participants $(27.7 \%)$ received simultaneous chemotherapy, whereas 19 (15.9\%) were treated with simultaneous radiotherapy. Different chemotherapy schedules were used, with the majority of them being platinum-based regimens.

\section{Sputum culture}

Expectorated sputum samples were collected in sterile specimen containers and transported and processed immediately in the hospital bacteriology laboratory. The technique of sample selection for Gram stain as well as cultures consisted of teasing out the purulent portion of the sputum, a section of which was then spread thinly over a glass slide. Gram stains were examined by trained personnel. All Gram stains were first screened under a mi-

Table 1. General characteristics of patients with lung cancer and pneumonia

\begin{tabular}{|c|c|}
\hline Variable & Study patients $(n=119)$ \\
\hline Male sex, $n(\%)$ & $111(93.3)$ \\
\hline Age (years) & $59.8 \pm 9.6$ \\
\hline Diabetes mellitus, $n$ (\%) & $13(10.9)$ \\
\hline COPD, $n(\%)$ & $11(9.2)$ \\
\hline $\begin{array}{l}\text { Histology, } n(\%) \\
\text { Squamous cell carcinoma } \\
\text { Adenocarcinoma } \\
\text { Small cell lung cancer }\end{array}$ & $\begin{array}{l}53(44.5) \\
51(42.8) \\
15(12.7)\end{array}$ \\
\hline $\begin{array}{l}\text { Tumour stage, } n(\%) \\
\qquad \begin{array}{l}1 \\
2 \\
3 \\
4 \\
\text { Unknown }\end{array}\end{array}$ & $\begin{array}{c}0(0) \\
13(10.9) \\
28(23.5) \\
71(59.7) \\
7(5.9)\end{array}$ \\
\hline $\begin{array}{l}\text { Tumour localisation, } n(\%) \\
\text { Central } \\
\text { Peripheral }\end{array}$ & $\begin{array}{l}44(36.9) \\
75(63.1)\end{array}$ \\
\hline Surgical treatment, $n$ (\%) & $26(21.8)$ \\
\hline Pleural effusion, $n$ (\%) & $25(21.0)$ \\
\hline Haemoglobin, g/l & $10.2(6.0-15.0)$ \\
\hline C-reactive protein, $\mathrm{mg} / \mathrm{dl}$ & $12.1(0.34-324.6)$ \\
\hline Creatinine, mg/dl & $0.8(0.4-1.9)$ \\
\hline White blood count, $\mathrm{mm}^{3}$ & $10,400(500-216,000)$ \\
\hline
\end{tabular}

croscope at low power to assess the appropriateness of the sample. Sputum samples were considered of good quality if they had numerous polymorphonuclear cells (> 25 in a 100 × microscopic field) and few squamous epithelial cells (< 10 in a $100 \times$ microscopic field). Otherwise, the sputum sample was considered to be contaminated with saliva and was rejected. A total of 20 samples were initially rejected because of saliva contamination, but a second sampling was successful in obtaining good quality sputum samples that were suitable for microbiological analyses. Good quality samples were then screened for a predominant morphological type in an oil immersion field. The presence of a predominant morphotype was considered when the Gram stain showed bacteria only or when it mainly corresponded to the gram morphotype revealed by standard microbiological criteria. Sputum cultures were processed immediately on blood agar, chocolate agar, and bromothymol blue lactose agar media. Growing isolates (fungi or bacteria) were identified according to routine bacteriological procedures. If first sputum was inadequete, the second sputum was taken.

\section{Treatment of pneumonia}

Empiric treatment was implemented in nine patients, and its appropriateness was subsequently evaluated by analysing the antibiogram of the isolated microorganisms. Appropriate treatment modifications were performed when necessary based on the results of definitive antibiogram. In the remaining patients $(n=110)$, treatment was directly informed by the results of antibiogram.

\section{Data analysis}

Statistical calculations were performed using SPSS release 17.0 (SPSS Inc., Chicago, IL, USA). Continuous data

Table 2. Multivariate analysis of having a positive Aspergillus fumigatus sputum culture in the study patients $(n=119)$

\begin{tabular}{|lcc|}
\hline Variable & $\begin{array}{c}\text { Odds ratio } \\
(95 \% \mathrm{Cl})\end{array}$ & $p$-value \\
\hline Male sex & $1.01(0.96-1.89)$ & NS \\
\hline Age & $1.21(0.73-2.13)$ & NS \\
\hline Diabetes mellitus & $1.34(0.91-1.67)$ & NS \\
\hline COPD & $1.98(0.97-2.45)$ & NS \\
\hline Histology & $0.86(0.59-1.06)$ & NS \\
\hline Tumour stage & $1.12(0.89-1.44)$ & NS \\
\hline Tumour localisation & $0.78(0.61-1.82)$ & NS \\
\hline Surgical treatment & $1.04(0.97-1.12)$ & NS \\
\hline Pleural effusion & $0.98(0.86-1.86)$ & NS \\
\hline Hospital-acquired pneumonia & $1.33(0.91-2.30)$ & NS \\
\hline Chemotherapy dose & $1.34(0.90-1.78)$ & NS \\
modifications & $1.11(0.87-1.95)$ & NS \\
\hline Respiratory failure & $1.32(1.17-3.68)$ & $<0.05$ \\
\hline Febrile neutropaenia & $1.78(1.37-4.12)$ & $<0.01$ \\
\hline $\begin{array}{l}\text { Development of pneumonia } \\
\text { within the first 10 days }\end{array}$ & & \\
\hline Cl - confidence interval; NS - not significant & & \\
\hline
\end{tabular}


were tested for normality using the Shapiro-Wilk test and expressed as mean \pm SD (if the distribution was normal) or medians with interquartile ranges (in the presence of skewed variables). Multivariate logistic regression analysis was used to identify the independent predictors of having a positive Aspergillus fumigatus sputum culture (dependent variable). The potential covariates (predictors) involved in the multivariate model included: male sex, age, diabetes mellitus, COPD, histology, tumour stage, tumour localisation, surgical treatment, pleural effusion, hospital-acquired pneumonia, chemotherapy dose modifications after the diagnosis of pneumonia, respiratory failure, febrile neutropaenia, and development of pneumonia within the first 10 days of chemotherapy initiation. The results of this analysis were expressed as adjusted odds ratios (ORs) with 95\% confidence intervals ( $\mathrm{Cls}$ ). A value of $p<0.05$ (two-sided) was considered statistically significant.

\section{Results}

\section{General characteristics and clinical course}

The general characteristics of the study participants are reported in Table 1 . Ten patients (8.4\%) had hospital-acquired pneumonia, whereas the remaining cases had CAP. A total of 75 patients (63\%) had received chemotherapy before developing pneumonia. Of them, 32 developed pneumonia within the first 10 days of chemotherapy initiation. In 10 patients chemotherapy doses were modified after the diagnosis of pneumonia. Febrile neutropaenia developed in 22 patients (18.5\%), of whom 21 received granulocyte colony-stimulating factor (G-CSF) treatment. Eight patients (6.8\%) received ventilatory support because of respiratory failure. At the time of analyses, 40 patients (33.6\%) were alive with no evidence of disease, 29 (24.4\%) were alive with sequelae, and 50 (42\%) died. The median time of hospitalisation was 12 days (interquartile range: 7-19 days).

\section{Infection spectrum}

Of the 119 study patients, 92 (77.3\%) had positive isolates from sputum cultures. The most frequent isolate from the sputum of lung cancer patients with pneumonia was Aspergillus fumigatus $(n=22)$, followed by Haemophilus influenzae $(n=13)$, Pseudomonas aeruginosa $(n=12)$, Klebsiella pneumoniae $(n=9)$, Acinectobacter baumannii $(n=9)$, Streptococcus pneumoniae $(n=8)$, Staphylococcus aureus $(n=6)$, Escherichia coli $(n=4)$, Staphylococcus epidermidis $(n=3)$, and Enterobacter cloacae $(n=3)$. In the remaining three positive cultures, the isolated pathogens were Proteus mirabilis, Moraxella catarrhalis, and Moraxella osloensis. Patients with coinfections were excluded from the study.

\section{Multivariate analysis}

The results of multivariate logistic regression analysis are presented in Table 2. The likelihood of having a positive Aspergillus fumigatus sputum culture was significantly and independently associated with febrile neutropaenia $(\mathrm{OR}=1.32,95 \% \mathrm{Cl}: 1.17-3.68, p<0.05)$ and the development of pneumonia within the first 10 days of chemotherapy initiation ( $\mathrm{OR}=1.78,95 \% \mathrm{Cl}: 1.37-4.12, p<0.01)$.

\section{Discussion}

This retrospective study was devised to investigate the microbiological spectrum of pathogens causing pneumonia in patients with lung cancer. In our series, Aspergillus fumigatus was the most frequent isolate, but the high diversity of pathogens clearly challenges the empirical use of antibiotics. Although empirical treatment is generally recommended for severe pulmonary infections in lung cancer $[8,9]$, our study highlights the need for a more rational use of antibiotic agents paired with improved education on the use of anti-infective drugs in patients with malignancies.

Lanoix et al. [11] previously investigated the microbiological characteristics of 87 lung cancer patients who developed febrile neutropaenia. The authors demonstrated that most of the identified bacteria in their series were germs of the urinary and digestive tracts (rather than respiratory germs) [11]. In addition, microbiologically documented fever was more likely to involve Gram-negative bacteria than Gram-positive bacteria. Conversely to the study by Lanoix et al. [11], in this report we specifically focused on lung cancer patients with pneumonia. Zięba et al. [9] previously demonstrated that pneumonia accounted for $58.5 \%$ of all deaths in a series of Polish patients with lung cancer. In a study by Putinati et al. [6], the frequency of occurrence of infections in lung cancer patients was estimated based on the results of the bacteriological examination of bronchoalveolar lavage fluid. By using this approach, an infectious agent was isolated in 34.3\% of patients, with Gram-negative rods being the most frequent cause of infections [6]. In line with these data, Remiszewski et al. [12] reported that the most frequent aetiological agents of infections resulting in the death of patients with lung cancer were Gram-negative bacteria. Although a high diversity of pathogens was found in the current study, Aspergillus fumigatus was predominant in the majority of our lung cancer cases with pneumonia. Previous studies have shown that Aspergillus fumigatus infections are common in immunocompromised patients, and that the lungs are the most common organ involved $[13,14]$. Notably, the likelihood of having a positive Aspergillus fumigatus sputum culture in our lung cancer patients was significantly and independently associated with febrile neutropaenia and the development of pneumonia within the first 10 days of chemotherapy initiation. Taken together, these results suggest that chemotherapy-induced neutropaenia and immunosuppression are important determinants of the excess risk of Aspergillus fumigatus pneumonia in patients with lung cancer. Other common causes of pneumonia in our study were Haemophilus influenzae and Pseudomonas aeruginosa. In general, Haemophilus influenzae is a leading cause of pneumonia in adults, especially in the presence of underlying lung disease (particularly chronic obstructive pulmonary disease [COPD]) [15]. Our data suggest that not only patients with COPD but also those with lung cancer are at high risk of developing Haemophilus influenzae pneumonia. Pseudomonas aeruginosa is a common human saprophyte that is ubiquitous in the environment and rarely causes disease in healthy people. 
However, it can cause serious infections in compromised individuals such as those with lung cancer, both because of chemotherapy-induced immunodeficiency and disrupted physical barriers [16].

Although our current findings are valuable in a setting (patients with lung cancer) where information on the infection spectrum of pneumonia are scarce, several limitations need to be highlighted. First, antimicrobial resistance patterns need to be investigated in detail in future studies. Second, the exact source of infection remains unclear. Third, data on postoperative chemotherapy were lacking. And fourth, the study participants were mostly males and further studies are needed to determine whether our results would apply equally to females. These caveats notwithstanding, the current study provides important data on the microbial spectra causing pneumonia in patients with lung cancer. When initiating empirical antimicrobial therapy, clinicians should also take into consideration the entire microbial spectrum.

The authors declare no conflict of interest.

\section{References}

1. Cakir Edis E, Karlikaya C. The cost of lung cancer in Turkey. Tuberk Toraks 2007; 55: 51-8.

2. Demirci E, Daloglu F, Gundogdu C, Calik M, Sipal S, Akgun M. Incidence and clinicopathologic features of primary lung cancer: a North-Eastern Anatolia region study in Turkey (2006-2012). Asian Pac J Cancer Prev 2013; 14: 1989-93.

3. Nichols L, Saunders R, Knollmann FD. Causes of death of patients with lung cancer. Arch Pathol Lab Med 2012; 136: 1552-7.

4. Nagata N, Nikaido Y, Kido M, Ishibashi T, Sueishi K. Terminal pulmonary infections in patients with lung cancer. Chest 1993; 103: 1739-42.

5. Perlin E, Bang KM, Shah A, et al. The impact of pulmonary infections on the survival of lung cancer patients. Cancer 1990; 66: 593-6.

6. Putinati S, Trevisani L, Gualandi M, et al. Pulmonary infections in lung cancer patients at diagnosis. Lung Cancer 1994; 11: 243-9.

7. Berghmans T, Sculier JP, Klastersky J. A prospective study of infections in lung cancer patients admitted to the hospital. Chest 2003; 124: 114-20.

8. Vento S, Cainelli F, Temesgen Z. Lung infections after cancer che motherapy. Lancet Oncol 2008; 9: 982-92.

9. Zięba M, Baranowska A, Krawczyk M, et al. Pneumonia as a cause of death in patients with lung cancer. Radiol Oncol 2003; 37: 167 74

10. Miyashita N, Shimizu H, Ouchi K, et al. Assessment of the usefulness of sputum Gram stain and culture for diagnosis of community-acquired pneumonia requiring hospitalization. Med Sci Monit 2008; 14: CR171-6.

11. Lanoix JP, Pluquet E, Lescure FX, et al. Bacterial infection profiles in lung cancer patients with febrile neutropenia. BMC Infect Dis 2011; 11: 183

12. Remiszewski P, Słodkowska J, Wiatr E, et al. Fatal infection in patients treated for small cell lung cancer in the Institute of Tuber culosis and Chest Diseases in the years 1980-1994. Lung Cancer 2001; 31: 101-10.

13. Latgé JP. The pathobiology of Aspergillus fumigatus. Trends Microbiol 2001; 9: 382-9.

14. Latgé JP. Aspergillus fumigatus and aspergillosis. Clin Microbiol Rev 1999; 12: 310-50.

15. Puig C, Calatayud L, Martí S, et al. Molecular epidemiology of nontypeable Haemophilus influenzae causing community-acquired pneumonia in adults. PLoS One 2013; 8: e82515.
16. Fujitani S, Sun HY, Yu VL, Weingarten JA. Pneumonia due to Pseudomonas aeruginosa: part I: epidemiology, clinical diagnosis, and source. Chest 2011; 139: 909-19.

\section{Address for correspondence}

Nilüfer Avcı

Ataruk state Turgut Reis St.

1000 Balikesir, Turkey

e-mail: drniluferavci@hotmail.com

Submitted: 23.06 .2014

Accepted: 31.10.2014 